\title{
Effect on Plasma Protein S Activity in Patients Receiving the Factor Xa Inhibitors
}

\author{
Takako Terakami ${ }^{1,2}$, Satomi Nagaya ${ }^{1}$, Kenshi Hayashi ${ }^{1}$, Hiroshi Furusho ${ }^{3}$, Noboru Fujino ${ }^{1,4}$, Takeshi Kato ${ }^{4}$, \\ Hidesaku Asakura ${ }^{5}$ and Eriko Morishita ${ }^{1,5}$
}

Takako Terakami and Satomi Nagaya contributed equally to this manuscript.

\author{
${ }^{1}$ Department of Clinical Laboratory Science, Division of Health Sciences, Graduate School of Medical Science, Kanazawa \\ University, Kanazawa, Japan \\ ${ }^{2}$ Department of Clinical Laboratory, Kanazawa University Hospital, Kanazawa, Japan \\ ${ }^{3}$ Department of Cardiology, Ishikawa Prefectural Central Hospital, Kanazawa, Japan \\ ${ }^{4}$ Department of Cardiology, Kanazawa University Hospital, Kanazawa, Japan \\ ${ }^{5}$ Department of Hematology, Kanazawa University Hospital, Kanazawa, Japan
}

Aims: Measurement of protein S (PS) activity in patients taking direct oral anticoagulants (DOACs) using reagents based on a clotting assay results in falsely high PS activity, thus masking inherited PS deficiency, which is most frequently seen in the Japanese population. In this study, we investigated the effect of factor Xa (FXa) inhibitors on PS activity using the reagent on the basis of the chromogenic assay, which was recently developed in Japan.

Methods: The study enrolled 152 patients ( 82 males and 70 females; the average age: $68.5 \pm 14.0$ years) receiving three FXa inhibitors (rivaroxaban, edoxaban, and apixaban). PS activity was measured using the reagents on the basis of the clotting and chromogenic assays.

Results: PS activity measured by the clotting assay reagents exhibited falsely high values depending on the plasma concentrations of FXa inhibitors in patients taking either rivaroxaban or edoxaban. However, none of the three FXa inhibitors affected PS activity when measured using the chromogenic assay.

Conclusion: In patients taking rivaroxaban or edoxaban, inherited PS deficiency is likely missed because the levels of PS activity measured using the reagents based on the clotting assay are falsely high. However, we report that three FXa inhibitors do not affect PS activity measured by the chromogenic assay. When measuring the levels of PS activity in patients undergoing DOACs, the principles of each reagent should be understood. Furthermore, plasma samples must be collected at the time when plasma concentrations of DOACs are lowest or the DOAC-Stop reagent should be used.

Key words: Factor Xa inhibitor, Inherited protein S deficiency, Protein S Tokushima, Clotting assay, Chromogenic assay

\section{Introduction}

Direct oral anticoagulants (DOACs) have been available since 2014 for the prevention of stroke caused by nonvalvular atrial fibrillation (AF) and for the treatment of venous thromboembolism (VTE), and their frequency of use continues to increase yearly ${ }^{1-5)}$. DOACs are classified as thrombin inhibitor (dabigatran) or factor $\mathrm{Xa}$ (FXa) inhibitors (rivaroxaban, edoxaban, and apixaban) based on the target of inhibition. Compared with vitamin $\mathrm{K}$ antagonists such as warfarin, DOACs exhibit more

Address for correspondence: Eriko Morishita, Department of Clinical Laboratory Science, Division of Health Sciences, Graduate School of Medical Science, Kanazawa University, 5-11-80 Kodatsuno, Kanazawa, Ishikawa 920-0942, Japan. E-mail: eriko86@staff.kanazawa-u.ac.jp

Received: April 5, $2021 \quad$ Accepted for publication: June 22, 2021

Copyright@2021 Japan Atherosclerosis Society

This article is distributed under the terms of the latest version of CC BY-NC-SA defined by the Creative Commons Attribution License. 


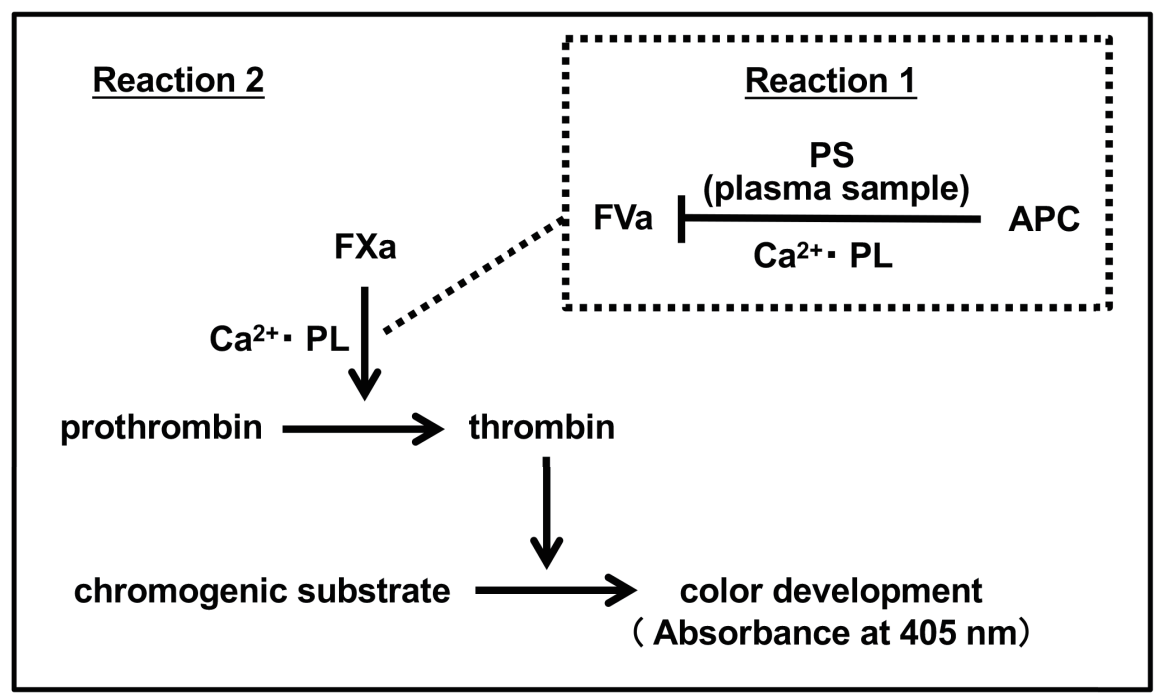

Fig. 1. Principle of total PS activity measurement using a chromogenic assay

PS in the plasma sample is reacted with APC and FVa for a certain period of time, and FVa is inactivated by PS (reaction 1). Residual FVa forms a prothrombinase complex with FXa and phospholipid and then activates prothrombin to thrombin. Since the generated thrombin degrades the chromogenic substrate, PS activity is indirectly detected by colorimetric quantification of its coloration (reaction 2).

predictable pharmacokinetic and pharmacodynamics profiles and therefore do not require routine coagulation monitoring ${ }^{()}$.

Thrombosis is caused by a combination of various environmental and genetic factors. Inherited thrombophilia is associated with a high risk of VTE and involves deficiencies in the natural anticoagulants: protein $S$ (PS), antithrombin (AT), and protein C $(\mathrm{PC})^{7,8)}$. Thus, the assays that measure the levels of PS, AT, and PC activities are essential screening tests. In Japan, inherited PS deficiency is the most common inherited thrombophilia, with an incidence of $1 \%-2 \%$ in the Japanese population, approximately 5-10 times higher than that $(0.16 \%-0.21 \%)$ in Europe and the United States ${ }^{9-12)}$. This higher incidence in Japan is associated with the presence of the PS Tokushima variant (PS p.Lys196Glu: PS K196E). The reported frequency of the PS K196E allele in the Japanese population is $0.9 \%^{9-11)}$, and PS K196E is considered a pathogenic variant in the Japanese population ${ }^{13,14)}$.

Inherited PS deficiency is classified internationally into three types and diagnosed primarily based on the antigen and activity levels of PS. PS K196E is classified as a type II PS deficiency, and the average PS activity in PS K196E heterozygotes is approximately $16 \%$ lower than that of wild-type individuals (PS K196), but this is not associated with a decrease in PS antigen levels. Furthermore, since the decrease in PS activity of PS K196E is mild (16\% decrease), even if PS activity is accurately measured,
PS K196E is likely to be missed ${ }^{15)}$.

Previous studies reported that patients taking DOACs exhibit falsely high PC and PS activity levels when measured using the reagents based on the clotting assay ${ }^{16-20)}$. We reported that this effect of FXa inhibitors can be avoided by measuring PC activity using the reagents based on the chromogenic assay ${ }^{21)}$. However, global guidelines recommended measuring the free PS antigen levels, not PS activity, in patients taking DOACs because there is no other method available for measuring PS activity other than the clotting assay reagents ${ }^{22)}$. Hence, a novel chromogenic assay for measuring total PS activity was recently developed in Japan ${ }^{23,24)}$.

Fig. 1 shows the principle of the chromogenic assay. In the first reaction, PS in plasma reacts with activated protein $\mathrm{C}(\mathrm{APC})$ and factor $\mathrm{Va}(\mathrm{FVa})$ in the reagent for a certain period of time, and then, APC inactivates FVa by using PS as a cofactor. Since the reagent contains a certain excess amount of $\mathrm{FVa}, \mathrm{FVa}$ remains after the inactivation of $\mathrm{FVa}$ in the first reaction. In the second reaction, the residual $\mathrm{FVa}$, together with FXa and phospholipid, activates prothrombin to thrombin, and the generated thrombin degrades the synthetic substrate, resulting in coloration. The PS activity is measured indirectly by colorimetric determination of the coloration. Since this method uses the degradation of the synthetic substrate, it is not affected by clotting time. Our data show that the reagent for measuring PS activity based 
on the chromogenic assay is not affected by FXa inhibitors. Furthermore, we report the results of the effect of FXa inhibitors on AT activity using several reagents.

\section{Aim}

In this study, we investigated the effect of FXa inhibitors on PS activity in patients receiving them measured using a chromogenic assay reagent. We also measured PS activity using several reagents based on the conventional clotting assay and compared the results with those of the chromogenic assay to determine how the different measurement principles of the reagents affect the analyses.

\section{Materials and Methods}

\section{Study Subjects}

DOACs targeted in this study included the FXa inhibitors: rivaroxaban, edoxaban, and apixaban. The subjects included two groups of 152 patients who attended outpatient clinics of the Departments of Cardiology or Hematology and were diagnosed with AF or VTE: (i) patients already undergoing anticoagulant treatment with FXa inhibitors at the time of study initiation and (ii) patients newly prescribed FXa inhibitor therapy. The study was approved by the University of Kanazawa Ethics Committee (approval no. 530-2). Written informed consent was obtained from all patients before enrollment. Patients with creatinine concentration $>$ $2.0 \mathrm{mg} / \mathrm{dL}$ and estimated glomerular filtration rate $<$ $30 \mathrm{~mL} / \mathrm{min} / 1.73 \mathrm{~m}^{2}$ (according to the CockroftGault equation) were excluded. Patients were also excluded if they had been diagnosed with inherited PS, AT, PC deficiency or antiphospholipid syndrome, acute thrombosis, severe liver dysfunction, or nephrotic syndrome.

\section{Methods}

Sample Collection and Evaluation of Patients on FXa Inhibitor Therapy

Blood samples were collected in tubes containing $3.2 \%$ sodium citrate within $2 \mathrm{~h}$ after oral administration of FXa inhibitor in patients already undergoing FXa inhibitor therapy. Samples were then immediately centrifuged to prepare platelet-poor plasma and the plasma samples were stored at $-80^{\circ} \mathrm{C}$. Plasma concentration of FXa inhibitor, prothrombin time (PT), activated partial thromboplastin time (APTT), PS activity (clotting assay and chromogenic assay), the total amount of PS antigen, and AT activity were measured for all samples.

Sample Collection and Evaluation of Patients Newly Prescribed Rivaroxaban

Blood samples were collected from seven patients newly started on rivaroxaban before as well as 2 and 4-8 weeks after initiation of treatment. Within $2 \mathrm{~h}$ after oral administration, the samples were collected and immediately centrifuged. Plasma samples were stored at $-80^{\circ} \mathrm{C}$ and evaluated the changes in plasma rivaroxaban concentration, PT, APTT, and PS activity (clotting assay and chromogenic assay) before and after rivaroxaban administration.

\section{Coagulation Tests}

a) Plasma Concentrations of FXa Inhibitor (Rivaroxaban, Edoxaban, and Apixaban)

For all samples collected from patients treated with FXa inhibitors, drug concentrations were estimated using the Biophen DiXaI assay (Hyphen BioMed, Neuville-sur-Oise, France) using appropriate FXa inhibitor calibrators. Assays were performed on the CS-5100 hemostasis system (Sysmex, Kobe, Japan).

\section{b) PT and APTT}

Laboratory-based PT and APTT were measured using each reagent (Thromborel S, Siemens, Marburg, Germany, and Actin FSL, Siemens, respectively) on the CS-5100 hemostasis system. The normal ranges for PT and APTT are 10.6-13.0 and 27.3-40.3 s.

\section{C) Ps Activity (Clotting Assay)}

Stago STA Staclot Protein S (Fujirebio Inc., Tokyo, Japan), Protein S Ac (Siemens), and HEMOCLOT Protein S (Hyphen BioMed) were used to evaluate PS activity before and after newly introduction of rivaroxaban. Additionally, Hemos IL PS-Clot (IL Japan, Tokyo, Japan) was used to evaluate PS activity in patients undergoing $\mathrm{FXa}$ inhibitor therapy. Assays were performed on the CS-5100 hemostasis system.

\section{d) PS Activity (Chromogenic Assay)}

Total PS activity was measured using the Total Protein S activity "Shino-Test" (Shino-Test Corp., Kanagawa, Japan). Fig. 1 illustrates the measurement principle of the chromogenic assay.

\section{e) PS Antigen}

PS antigen level was measured using Cygnus Auto Total Protein S mass (Shino-Test Corp.) on the basis of a latex photometric immunoassay. 


\section{f) AT Activity}

AT activity was measured by the chromogenic assay reagents using Berichrom AT III (Thrombinbased assay, Siemens) and Testzym S AT III (Xa-based assay, Sekisui Medical, Tokyo, Japan).

\section{Statistical Analyses}

Statistical analyses were performed using the STAT VIEW program (Abacus Concepts, Berkeley, CA, USA). The Pearson product-moment correlation coefficient was used to evaluate the correlation between the levels of activity and antigen of natural anticoagulants assessed with various reagents. $P<0.05$ was considered indicative of statistical significance.

\section{Results}

Study Subjects (Patients Undergoing FXa Inhibitor Therapy)

A total of 152 patients ( 82 males and 70 females) with AF or VTE were on anticoagulant therapy with $\mathrm{FXa}$ inhibitors. The average age $( \pm \mathrm{SD})$ of patients was $68.5 \pm 14.0$ years. Table 1 summarizes the distribution of diseases among the patients. Of 123 patients with $\mathrm{AF}, 69$ had $\mathrm{AF}$ alone, and 38 had $\mathrm{AF}$ with chronic heart failure (CHF). Of the 38 patients with CHF, 22 also had other diseases (14 patients had sick sinus syndrome, and eight patients had atherothrombotic cerebral infarction), and the remaining 16 patients also had hypertrophic cardiomyopathy (five also with deep vein thrombosis). The diseases for which there were three or fewer patients are omitted from Table 1 .

Effect of Rivaroxaban, Edoxaban, and Apixaban on PT and APTT

We also examined the effects of plasma FXa inhibitors on PT and APTT. Both PT and APTT were significantly prolonged in a dose-dependent manner in patients taking rivaroxaban or edoxaban (Fig. 2A, 2B, 2D, and 2E). The greatest prolongation of PT was observed with edoxaban as compared with other drugs, and it prolonged PT to a greater degree than APTT (Fig. 2B; r $=0.838, P<0.001$ ). Similarly, the greatest prolongation of APTT was observed with rivaroxaban as compared with other drugs, and it prolonged APTT to a greater degree than PT (Fig. 2D; $r=0.851, P<0.001$ ). By contrast, apixaban had no effect on either PT (Fig.2C; r $=0.158$, $P=0.396$ ) or APTT (Fig. $2 F ; r=0.404, P=0.062$ ) at any plasma concentration examined.
Table 1. Characteristics of patients' diseases and complications

\begin{tabular}{lc}
\hline Disease & Cases \\
\hline Atrial fibrillation (AF) & Total 123 \\
AF & 69 \\
AF \& CHF & 16 \\
AF \& Hypertrophic cardiomyopathy & 16 \\
AF, CHF \& Sick sinus syndrome & 14 \\
AF, CHF \& Atherothrombotic cerebral infarction & 8 \\
VTE & Total 34 \\
DVT & 28 \\
DVT \& AF & 5 \\
Retinal vein thrombosis & 1 \\
\hline
\end{tabular}

Of the 123 patients with atrial fibrillation, if the number of patients with other comorbidities was less than 3 , they were not listed.

Effect of Rivaroxaban, Edoxaban, and Apixaban on PS Activity

a) Clotting Assay (Fig. 3, Open Circles)

PS activity was measured using the clotting assay reagents. Both rivaroxaban and edoxaban increased PS activity in a dose-dependent manner (rivaroxaban: $\mathrm{r}=0.792, P=0.0001$; edoxaban: $\mathrm{r}=0.616, P=0.002)$ (Fig. 3A, 3B). By contrast, apixaban had no effect on $P S$ activity $(\mathrm{r}=0.037, P=0.909)$ (Fig. 3C).

\section{b) Chromogenic Assay (Fig. 3, Closed Circles)}

None of the three FXa inhibitors affected PS activity when measured using the chromogenic assay reagent (Fig.3A-C).

Effect of Rivaroxaban, Edoxaban, and Apixaban on PS Antigen (Fig. 3, Closed Triangles)

None of the three FXa inhibitors had any effect on the amount of PS antigen based on the principle of the latex photometric immunoassay (Fig.3D-F).

Changes in PT, APTT, PS Activity, and Plasma Rivaroxaban Concentration before and after Rivaroxaban Introduction

Blood samples were collected before, as well as 2 and 4-8 weeks after rivaroxaban introduction in seven sequential patients. As shown in Fig.4, at each blood collection point, the plasma concentration of rivaroxaban was approximately $50-450 \mathrm{ng} / \mathrm{mL} 2 \mathrm{~h}$ after drug administration (Fig.4A). PT and APTT were clearly prolonged after administration compared with baseline before rivaroxaban administration (Fig. 4B, 4C).

Additionally, PS activity was significantly higher compared with baseline, as determined using the clotting assay reagents (Fig.4D). Interference was more pronounced with Protein S Ac (Siemens) and 
A. Rivaroxaban

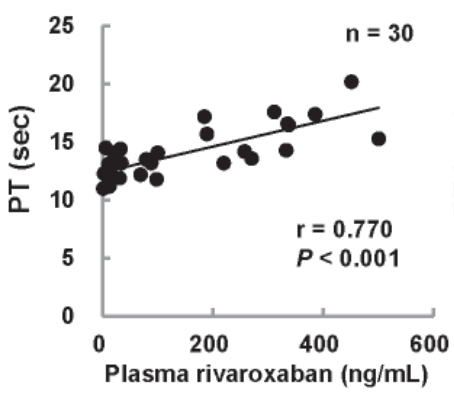

D. Rivaroxaban

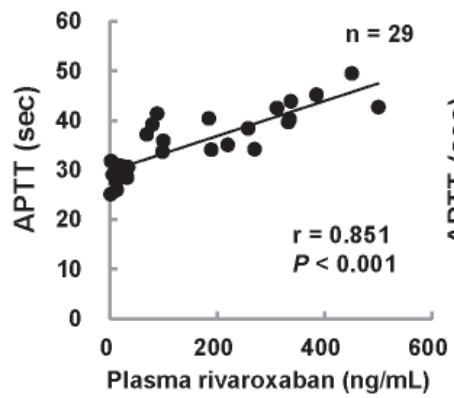

B. Edoxaban

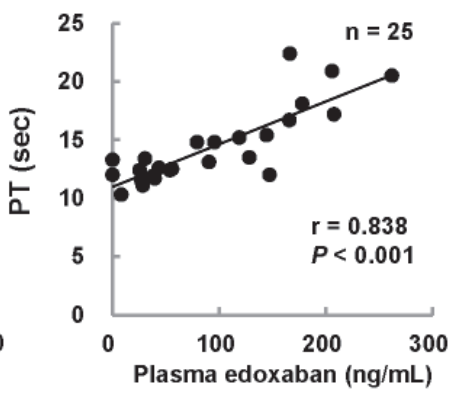

E. Edoxaban

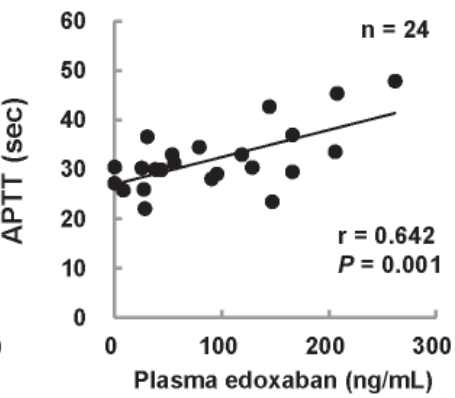

C. Apixaban

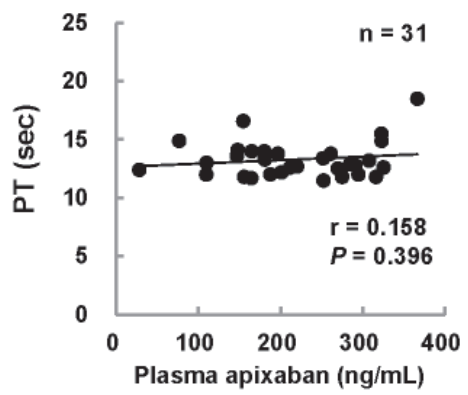

F. Apixaban

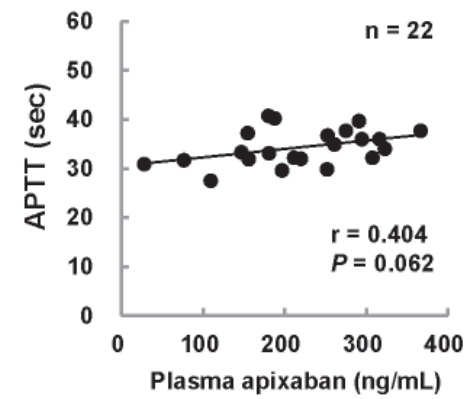

Fig. 2. Effect of rivaroxaban, edoxaban, and apixaban on PT and APTT A, D: Rivaroxaban, B, E: Edoxaban, C, F: Apixaban

A. Rivaroxaban

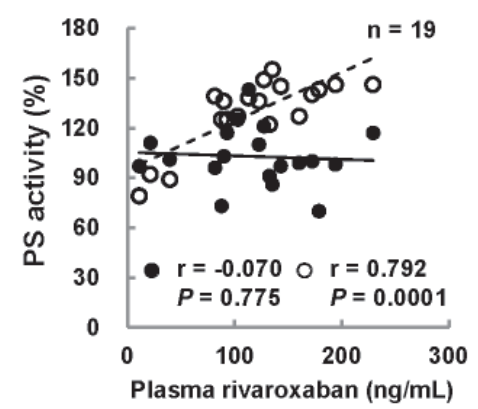

D. Rivaroxaban

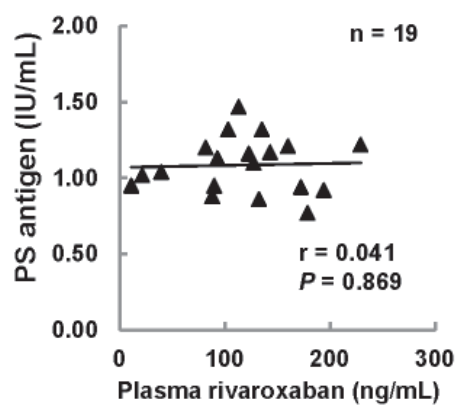

B. Edoxaban

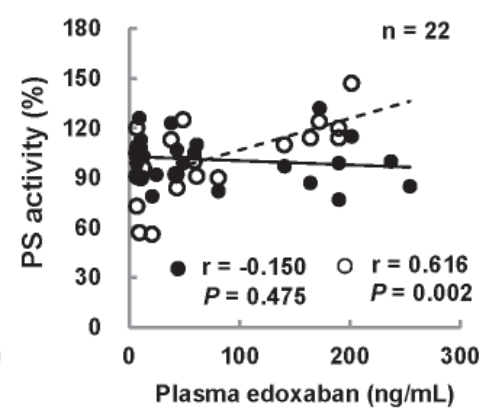

E. Edoxaban

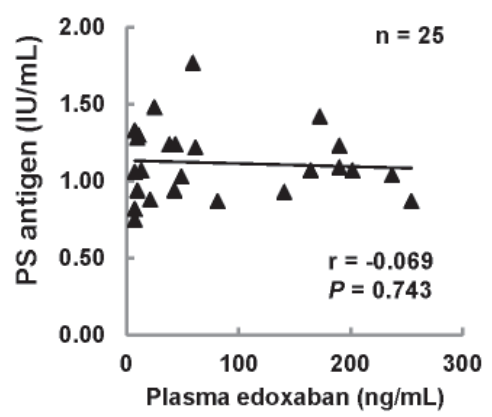

C. Apixaban

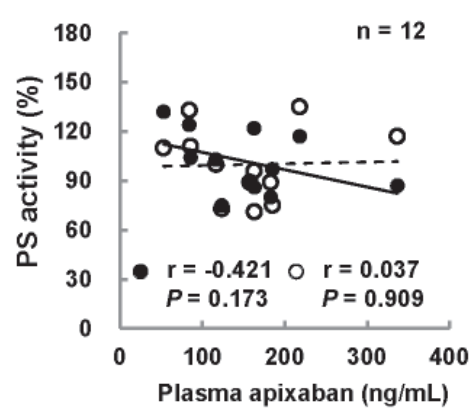

F. Apixaban

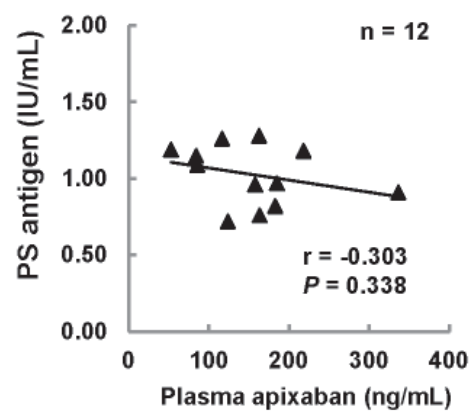

Fig. 3. Effect of rivaroxaban, edoxaban, and apixaban on the levels of PS activity and PS antigen

The graphs indicated PS activity measured using Chromogenic assay $(\mathbf{O})$ and Clotting assay $(\bigcirc)$, and PS antigen $(\boldsymbol{\Delta})$. A, D: Rivaroxaban, B, E: Edoxaban, C, F: Apixaban.

\section{Advance Publication Journal of Atherosclerosis and Thrombosis}




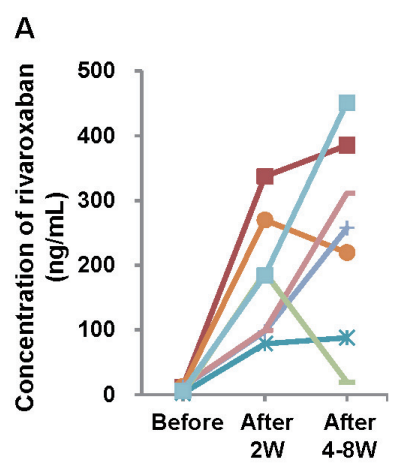

B

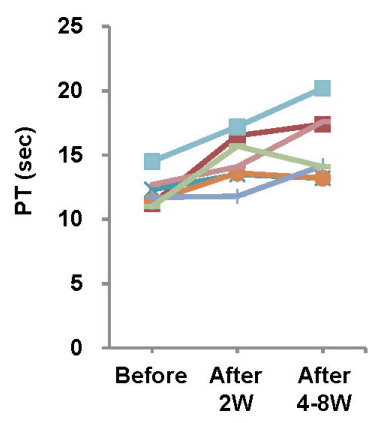

C

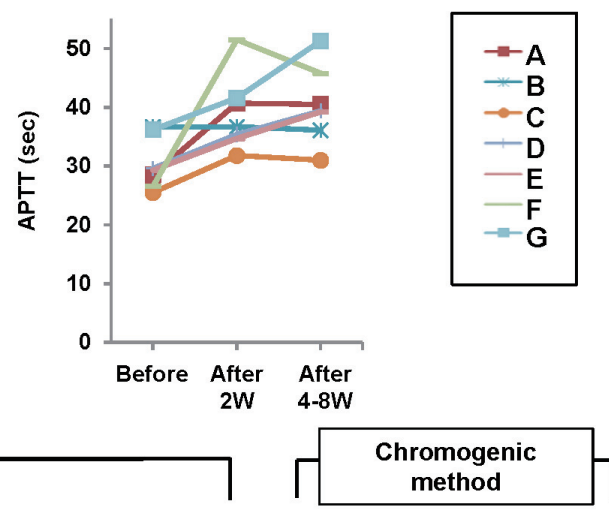

Shino-Test

PS total activity
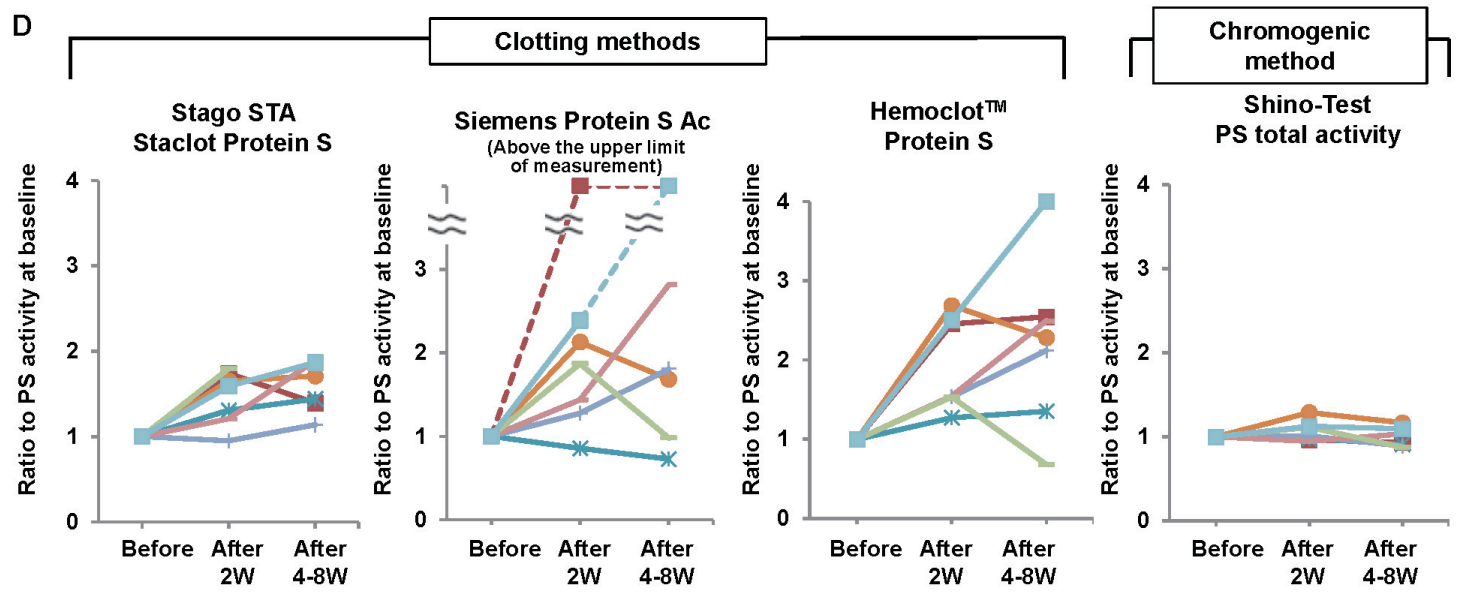

Fig.4. Plasma concentration of rivaroxaban and the changes in PT, APTT, and PS activity before and after rivaroxaban introduction

The graphs indicated the concentration of rivaroxaban (A), the change of PT (B) and APTT (C), and the changes in PS activity values by clotting assays or chromogenic assay before and after rivaroxaban introduction (D). "Stago STA Staclot Protein S," "Siemens Protein S Ac," and "HemoclotTM Protein S" are the clotting assay reagents, and "Shino-Test PS total activity" is the reagent based on the chromogenic assay.

HEMOCLOT Protein S (Hyphen BioMed). By contrast, little effect on PS activity was observed after rivaroxaban administration when measured using the chromogenic assay reagent (Fig.4D).

\section{Effect of Rivaroxaban, Edoxaban, and Apixaban on} AT Activity a) Thrombin-Based Assay (Fig. 5, Closed Squares)

None of the three FXa inhibitors affected AT activity when measured using the thrombin-based assay with a chromogenic method (Fig. 5A-C) ${ }^{19,25-27)}$.

\section{b) Xa-Based Assay (Fig. 5, Open Squares)}

All three FXa inhibitors increased AT activity in a dose-dependent manner, as measured by the Xa-based assay using a chromogenic method (Fig. 5AC; rivaroxaban, $\mathrm{r}=0.844, P<0.0001$; edoxaban, $\mathrm{r}=0.581, P=0.002$; apixaban, $\mathrm{r}=0.596, P=0.0003)$. Rivaroxaban had a particularly marked effect on AT activity $^{19,25-27)}$.

\section{Discussion}

The frequency of using DOACs has increased along with the increase in the number of patients with $\mathrm{AF}$ and VTE. DOACs have also been reported to affect the measurement of the activity of natural anticoagulants as well as the common coagulation tests, PT and APTT, which can lead to diagnostic errors.

The present study investigated the effect of FXa inhibitor administration on PS activity using two different methods, the clotting assay, and the chromogenic assay. In measurements of PS activity using the clotting assay, inactivation of FVa by APC in the reagent is detected as a prolongation of PT, with PS in the sample serving as a cofactor. Thus, DOACassociated prolongation of PT can lead to overestimation of plasma PS activity, resulting in falsely high values ${ }^{18,19,28)}$. By comparison, the chromogenic assay is not affected by clotting time because the chromogenic substrate is degraded, resulting in a color change that corresponds to the final thrombin activity 
A. Rivaroxaban

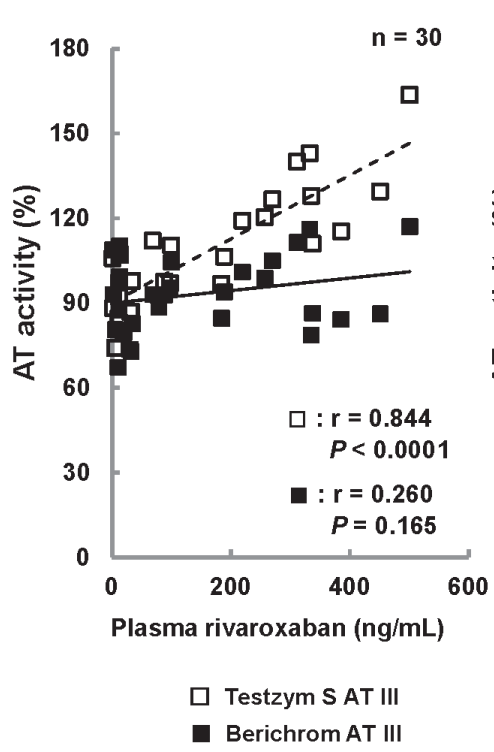

B. Edoxaban

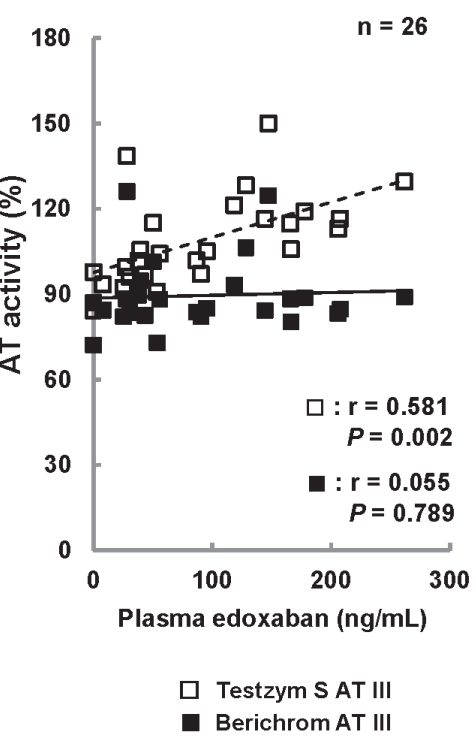

C. Apixaban

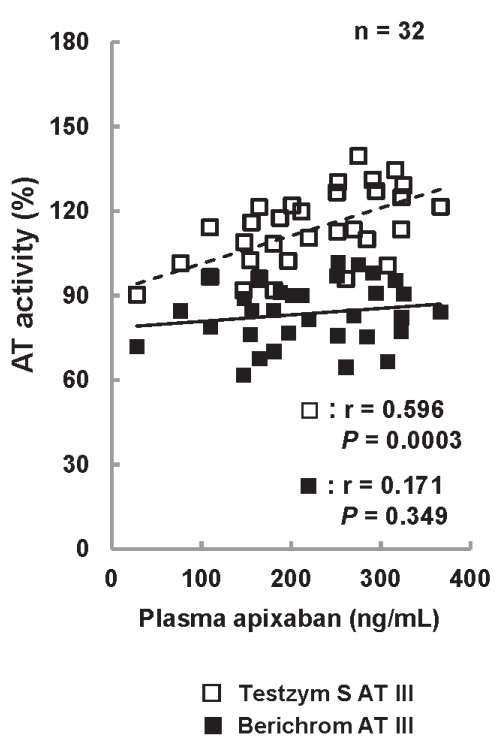

Fig. 5. Effect of rivaroxaban, edoxaban, and apixaban on AT activity

A: Rivaroxaban, B: Edoxaban, C: Apixaban, $\square$ : Xa-based assay,

: Thrombin-based assay

produced; PS activity is subsequently calculated by colorimetric determination ${ }^{23,24)}$ (Fig. 1). In samples from patients taking FXa inhibitors, significant prolongations of both PT and APTT were observed with increasing plasma concentrations of rivaroxaban and edoxaban. Apixaban had no such effect, however (Fig.2). Before our study, Kuma et al. reported that rivaroxaban and edoxaban prolonged PT and APTT in a drug concentration-dependent manner, whereas apixaban had no such effect in vitro experiments, and these results were similar to ours ${ }^{29)}$. However, the study by Kuma et al. differs from our study because they did not use patients' plasma samples. The prolongation of clotting time observed in this study could be due to physical or chemical interactions between the individual $\mathrm{FXa}$ inhibitors and phosphatidylserine present in the reagents. Additionally, it has been reported that the anti-FXa activity of apixaban at the peak plasma concentrations was only approximately $60 \%$ of that of rivaroxaban ${ }^{30,31)}$, so the difference in anti-FXa activity at the time of blood collection may have appeared as a difference in PT prolongation effect.

PS activity measured using the clotting assay depended on the concentrations of rivaroxaban and edoxaban and exhibited a significant positive correlation (Fig. 3A, 3B). Again, however, apixaban exhibited no such effect (Fig.3C). This result was consistent with the observation that apixaban has little effect on the measurement of PS activity by clotting assay, as PT was not prolonged in patients taking apixaban. In a previous study, FXa inhibitors caused falsely high PS activity when measured using the clotting assay ${ }^{16-19)}$. However, in the present study, only two drugs, rivaroxaban and edoxaban, increased PS activity in a drug concentration-dependent manner, perhaps because of the differences in reactivity with phosphatidylserine in the reagents used and in antiFXa activity in blood at the time of blood collection. By contrast, none of the three FXa inhibitors affected PS activity as measured using the chromogenic assay (Fig. 3A-C, closed circles). Similarly, none of the three FXa inhibitors affected the amount of total PS antigen as determined using the latex photometric immunoassay (Fig.3D-F).

We also evaluated the PT, APTT, and PS activity in patients before and after rivaroxaban introduction to confirm that the changes in clotting time and PS activity were associated with the introduction of rivaroxaban. These analyses showed that PT and APTT were significantly prolonged after the introduction of rivaroxaban (Fig.4B, 4C). Additionally, PS activity measured using the clotting assay correlated well with these prolongations of clotting time and exhibited falsely high values (Fig.4D). However, PS activity measured using the chromogenic assay did not otherwise change before and after rivaroxaban introduction. Hence, FXa inhibitors had no effect on PS activity measured using the reagent based on the chromogenic assay newly 
Table 2. Effect of DOACs on coagulation testing for inherited thrombophilia

\begin{tabular}{|c|c|c|c|c|c|c|}
\hline & \multirow{2}{*}{\multicolumn{2}{|c|}{ Methods }} & Thrombin & \multicolumn{3}{|c|}{ Factor $\mathrm{Xa}$ inhibitors } \\
\hline & & & Dabigatran & Rivaroxaban & Edoxaban & Apixaban \\
\hline \multirow[t]{2}{*}{ PS activity } & \multicolumn{2}{|l|}{ Chromogenic assay } & no effect ${ }^{*}$ & no effect & no effect & no effect \\
\hline & \multicolumn{2}{|l|}{ Clotting assay } & false high $^{*}$ & false high & false high & no effect \\
\hline \multirow[t]{2}{*}{ AT activity } & \multirow[t]{2}{*}{ Chromogenic assay } & Thrombin-based assay & false high ${ }^{*}$ & no effect & no effect & no effect \\
\hline & & Xa-based assay & no effect ${ }^{*}$ & false high & false high & false high \\
\hline
\end{tabular}

developed in Japan. Thus, PS activity should be measured using the chromogenic assay to accurate measurement during FXa inhibitor treatment.

AT activity is measured using the reagents on the basis of the chromogenic assay, of which there are two types: thrombin-based assay and Xa-based assay. AT activity measured using the thrombin-based assay was not significantly correlated with plasma concentration for any of the FXa inhibitors examined. By contrast, AT activity measured using the Xa-based assay was positively correlated with plasma concentration for all three FXa inhibitors, with rivaroxaban having the greatest effect on AT activity (Fig. 5). In the Xa-based assay, the FXa inhibitor present in the blood inhibits FXa contained in the reagent, which is thought to reduce the residual FXa activity, resulting in falsely high AT activity ${ }^{16-19,25-27)}$. The different effects of FXa inhibitors on AT activity could be related to differences in reactivity regarding the FXa inhibitory mechanism of each drug. In the present study, similar to the previous reports $16,18,19,25-27)$, AT activity in patients taking FXa inhibitors was unaffected by the FXa inhibitors when measured using the thrombinbased assay reagents.

The anticoagulant effects of various DOACs interfere with tests that measure coagulation activity in patient plasma samples. Thus, the screening tests for inherited thrombophilia in patients undergoing DOAC therapy may show falsely high PS, AT, and PC activity. It is desirable to stop DOAC therapy before coagulation testing, but in practice, it is difficult to stop administration once it has been started for the sole purpose of testing. Collecting blood samples during the trough period, when the plasma concentration of DOAC is as low as possible, is one way to minimize interference ${ }^{19,}{ }^{32)}$. Presently, coagulation testing can be performed without DOAC interference using the "DOAC Stop" reagent, which neutralizes the anticoagulant effect of DOACs in blood $^{33,34)}$, but the high cost of this reagent can be prohibitive.

Table 2 summarizes the results of this study. The chromogenic assay developed in Japan was shown to be suitable for measuring PS activity, which can lead to the identification of inherited PS deficiency. Although the effect of thrombin inhibitor was not investigated in this study, PS and PC activity measured using the clotting assay may be falsely high, as the anticoagulant effect of dabigatran is reflected in the APTT $\left.{ }^{16}, 18,19,32,35,37\right)$. By contrast, thrombin inhibitor as well as FXa inhibitors is presumed to have no effect on PS and PC activity measured using the chromogenic assay ${ }^{16,18,19)}$. The differences of DOACspecific reactivity in the reagents of natural anticoagulant activity could be due to characteristics of DOAC, such as their pharmacologic activity, mechanism of $\mathrm{FXa}$ or thrombin inhibition, and reactivity to assay reagents, but this is not clear and should be investigated in further studies ${ }^{19-21,32,38,39)}$.

\section{Conclusion}

Measurements of the activity of various natural anticoagulants (PS, AT, and PC) in patients taking FXa inhibitors are affected by the type of DOAC and the principle of the assay reagent. Additionally, reagents with the same measurement principle can exert different effects on the measured values. In this study, we found that a newly developed reagent for measuring PS activity based on a chromogenic method in Japan was not affected by FXa inhibitors. In the future, this chromogenic method will be used to measure PS activity for rapid and accurate diagnosis of inherited PS deficiencies such as the PS K196E allele variant. Screening for inherited thrombophilia in patients undergoing DOAC therapy requires

\section{Advance Publication Journal of Atherosclerosis and Thrombosis}


familiarity with the principles and characteristics of the assay reagents as well as parameters specific to DOACs. Furthermore, it is important to control the effect on coagulation tests of these drugs by collecting blood at the appropriate time, such as during the trough phase or by using the DOAC Stop reagent.

\section{Conflicts of Interest} disclose.

All authors have no conflicts of interest to

\section{Acknowledgments}

We would like to express our sincere gratitude to the patients who participated in our study and to Tsukasa Suetake (Sysmex, Kobe, Japan), Takeshi Suzuki (Sysmex), Satoka Kohama (Sysmex), and Tomohide Tsuda (Shino-Test Corporation, Kanagawa, Japan) for technical assistance with the experiments. This work was partially supported by a Grant-in-Aid from the Ministry of Health, Labor and Welfare of Japan to EM (20FC1024).

\section{References}

1) Castellucci LA, Cameron C, Le Gal G, Rodger MA., Coyle D, Wells PS, Clifford T, Gandara E, Wells G, Carrier M: Clinical and safety outcomes associated with treatment of acute venous thromboembolism: a systematic review and meta-analysis. JAMA, 2014; 312: 1122-1135

2) Verhamme P, Wells PS, Segers A, Ageno W, Brekelmans MP, Cohen AT, Meyer G, Grosso MA, Raskob G, Weitz JI, Zhang G, Buller H: Dose reduction of edoxaban preserves efficacy and safety for the treatment of venous thromboembolism. An analysis of the randomised, double-blind HOKUSAI VTE trial. Thromb Haemost, 2016; 116: 747-753

3) Almutairi AR, Zhou L, Gellad WF, Lee JK, Slack MK, Martin JR, Lo-Ciganic W-H: Effectiveness and Safety of Non-vitamin K Antagonist Oral Anticoagulants for Atrial Fibrillation and Venous Thromboembolism: A Systematic Review and Meta-analyses. Clin Ther, 2017; 39: 14561478

4) Nick van Es, Coppens M, Schulman S, Middeldorp S, Büller HR: Direct oral anticoagulants compared with vitamin $\mathrm{K}$ antagonists for acute venous thromboembolism: evidence from phase 3 trials. Blood, 2014; 124: 19681975

5) Forster R, Stewart M: Anticoagulants (extended duration) for prevention of venous thromboembolism following total hip or knee replacement or hip fracture repair. Cochrane Database Syst Rev, 2016; Mar 30; 3: CD004179

6) Eikelboom JW, Quinlan DJ, Hirsh J, Connolly SJ, Weitz JI: Laboratory monitoring of non-vitamin $\mathrm{K}$ antagonist oral anticoagulant use in patients with atrial fibrillation: a review. JAMA Cardiol, 2017; 2: 566-574

7) Miyata T, Sato Y, Ishikawa J, Okada H, Takeshita S, Sakata T, Kokame K, Kimura R, Honda S, Kawasaki T, Suehisa E, Tsuji H, Madoiwa S, Sakata Y, Kojima T, Murata M, Ikeda Y: Prevalence of genetic mutations in protein $S$, protein $\mathrm{C}$ and antithrombin genes in Japanese patients with deep vein thrombosis. Thromb Res, 2009; 124: $14-18$

8) Patracchini P, Aiello V, Palazzi P, Calzolari E, Bernardi F: Sublocalization of the human protein $\mathrm{C}$ gene on chromosome 2q13-q14. Hum Genet, 1989; 81: 191-192

9) Kinoshita $S$, Iida $H$, Inoue $S$, Watanabe $K$, Kurihara $M$, Wada Y, Tsuda H, Kang D, Hamasaki N: Protein S and protein $\mathrm{C}$ gene mutation in Japanese deep vein thrombosis patients. Clin Biochem, 2005; 38: 908-915

10) Tsuda H, Noguchi K, Oh D, Bereczky Z, Lee LH, Kang D, Dusse LMS, Carvalho MG, Morishita E: SSC Subcommittee on Plasma Coagulation Inhibitors of the ISTH. Racial differences in protein S Tokushima and two protein $\mathrm{C}$ variants as genetic risk factors for venous thromboembolism. Res Pract Thromb Haemost, 2020; 4: 1295-1300

11) Hamasaki N, Kuma H, Tsuda $H$ : Activated protein $C$ anticoagulant system dysfunction and thrombophilia in Asia. Ann Lab Med, 2013; 33: 8-13

12) Sakata T, Okamoto A, Mannami T, Tomoike H, Miyata T: Prevalence of protein $S$ deficiency in the Japanese general population: the Suita Study. J Thromb Haemost, 2004; 2 : 1012-1013

13) Pecheniuk NM, Elias DJ, Xu X, Griffin JH: Failure to validate association of gene polymorphisms in EPCR, PAR-1, FSAP and protein S Tokushima with venous thromboembolism among Californians of European ancestry. Thromb Haemost, 2008; 99: 453-455

14) Liu W, Yin T, Okuda H, Harada KH, Li Y, Xu B, Yang J, Wang H, Fan X, Koizumi A, Miyata T: Protein S K196E mutation, a genetic risk factor for venous thromboembolism, is limited to Japanese. Thromb Res, 2013; 132: 314-315

15) Kimura R, Sakata T, Kokubo Y, Okamoto A, Okayama A, Tomoike H, Miyata T: Plasma protein S activity correlates with protein $S$ genotype but is not sensitive to identify K196E mutant carriers. J Thromb Haemost, 2006; 4: 2010-2013

16) Favaloro EJ, Lippi G: Laboratory testing in the era of direct or non-vitamin $\mathrm{K}$ antagonist oral anticoagulants: a practical guide to measuring their activity and avoiding diagnostic errors. Semin Thromb Hemost, 2015; 41: 208227

17) Douxfils J, Chatelain B, Chatelain C, Dogné JM, Mullier F: Edoxaban: Impact on routine and specific coagulation assays. A practical laboratory guide. Thromb Haemost, 2016; 115: 368-381

18) Gosselin RC, Adcock DM: The laboratory's 2015 perspective on direct oral anticoagulant testing. J Thromb Haemost, 2016; 14: 886-893

19) Douxfils J, Ageno W, Samama CM, Lessire S, Ten Cate H, Verhamme P, Dogné JM, Mullier F: Laboratory testing in patients treated with direct oral anticoagulants: A practical guide for clinicians. J Thromb Haemost, 2018; 16: 209-219 
20) Harenberg J, Du S, Weiss C, Krämer R, Hoppensteadt D, Walenga J: Report of the Subcommittee on Control of Anticoagulation on the determination of the anticoagulant effects of apixaban: communication from the SSC of the ISTH. J Thromb Haemost, 2014; 12: 801-804

21) Terakami T, Sekiya A, Hayashi K, Suzuki T, Furusho H, Asakura H, Morishita E, Wada T: The effect of direct oral anticoagulants on blood protein $\mathrm{C}$ activity. Journal of Wellness and Health Care, 2020; 44: 33-41

22) Brinkman HJM, Ahnström J, Castoldi E, Dahlbäck B, Marlar RA: Pleiotropic anticoagulant functions of protein $S$, consequences for the clinical laboratory. Communication from the SSC of the ISTH. J Thromb Haemost, 2021; 19: 281-286

23) Tsuda T, Jin X, Tsuda H, Ieko M, Morishita E, Adachi T, Hamasaki N: New quantitative total protein S-assay system for diagnosing protein $S$ type II deficiency: clinical application of the screening system for protein $S$ type II deficiency. Blood Coagul Fibrinolysis, 2012; 23: 56-63

24) Tsuda T, Jin X, Yoshimura H: Development of novel methods and reagents for measuring protein $S$-Assay systems for total protein $S$ activity, total protein $S$ mass and protein S specific activity-. Jpn J Clin Chem, 2019; 48: 245-251

25) Hillarp A, Baghaei F, Fagerberg B, Gustafsson KM, Stigendal L, Sten-Linder M, Strandberg K, Lindahl TL: Effects of the oral, direct factor Xa inhibitor rivaroxaban on commonly used coagulation assays. J Thromb Haemost, 2011; 9: 133-139

26) Van Blerk M, Bailleul E, Chatelain B, Demulder A, Devreese K, Douxfils J, Jochmans K, Mullier F, Wijns W, Soumali MR, Coucke W, Vernelen K, Van de Walle P: Influence of dabigatran and rivaroxaban on routine coagulation assays. A nationwide Belgian survey. Thromb Haemost, 2015; 113: 154-164

27) Mani H, Hesse C, Stratmann G, Lindhoff-Last E: Rivaroxaban differentially influences ex vivo global coagulation assays based on the administration time. Thromb Haemost, 2011; 106: 156-164

28) Bonar R, Favaloro EJ, Mohammed S, Ahuja M, Pasalic L, Sioufi J, Marsden K: The effect of the direct factor Xa inhibitors apixaban and rivaroxaban on haemostasis tests: a comprehensive assessment using in vitro and ex vivo samples. Pathology, 2016; 48: 60-71

29) Kuma H, Matsuda R, Nakashima A, Motoyama K, Takazaki S, Hatae H, Jin X, Tsuda T, Tsuda H, Hamasaki
N: Protein S-specific activity assay system is not affected by direct oral anticoagulants. Thromb Res, 2018; 168: 60-62

30) Ieko M: Characteristics of various anticoagulants and evaluation methods for risk of bleeding and thrombosis. Jpn J Electrocardiology, 2014; 34: 149-156 (in Japanese)

31) Frost C, Song Y, Barrett YC, Wang J, Pursley J, Boyd RA, LaCreta F: A randomized direct comparison of the pharmacokinetics and pharmacodynamics of apixaban and rivaroxaban. Clin Pharmacol, 2014; 6: 179-187

32) Samuelson BT, Cuker A, Siegal DM, Crowther M, Garcia DA: Laboratory assessment of the anticoagulant activity of direct oral anticoagulants: A systematic review. Chest, 2017; 151: 127-138

33) Platton S, Hunt C: Influence of DOAC Stop on coagulation assays in samples from patients on rivaroxaban or apixaban. Int J Lab Hematol, 2019; 41: 227-233

34) Favresse J, Lardinois B, Sabor L, Devalet B, Vandepapeliere J, Braibant M, Lessire S, Chatelain B, Jacqmin H, Douxfils J, Mullier F: Evaluation of the DOAC-Stop ${ }^{\circledR}$ procedure to overcome the effect of DOACs on several thrombophilia screening tests. TH Open, 2018; 2: e202-e209

35) Halbmayer WM, Weigel G, Quehenberger P, Tomasits J, Haushofer AC, Aspoeck G, Loacker L, Schnapka-Koepf M, Goebel G, Griesmacher A: Interference of the new oral anticoagulant dabigatran with frequently used coagulation tests. Clin Chem Lab Med, 2012; 50: 16011605

36) Lindahl TL, Banghaei F, Blixter IF, Gustafsson KM: Expert Group on Coagulation of the External Quality Assurance in Laboratory Medicine in Sweden: Effect of the oral, direct thrombin inhibitor dabigatran on five common coagulation assays. Thromb Haemost, 2011; 105: $371-378$

37) Douxfils J, Mullier F, Robert S, Chatelain C, Chatelain B, Dogné JM: Impact of dabigatran on a large panel of dabigatran etexilate. Thromb Haemost, 2012; 107: 985997

38) Harenberg, J, Marx S, Erdle S, Krämer R: Determination of the anticoagulant effects of new oral anticoagulants: an unmet need. Expert Rev Hematol, 2012; 5: 107-113

39) Lippi G, Favaloro EJ: Recent guidelines and recommendations for laboratory assessment of the direct oral anticoagulants (DOACs): is there consensus? Clin Chem Lab Med, 2015; 53: 185-197 